\title{
Médiévales
}

Langues, Textes, Histoire

47 | automne 2004

Îles du Moyen Âge

\section{Boccace, « de Canarie et d'autres îles nouvellement découvertes dans l'Océan, au large de l'Espagne »}

Traduction du latin et commentaire

\section{Nathalie Bouloux et Odile Redon}

\section{(2) OpenEdition}

\section{Journals}

Édition électronique

URL : https://journals.openedition.org/medievales/1598

DOI : 10.4000/medievales.1598

ISSN : 1777-5892

\section{Éditeur}

Presses universitaires de Vincennes

Édition imprimée

Date de publication : 1 décembre 2004

Pagination : $9-16$

ISBN : 2-84292-157-7

ISSN : 0751-2708

\section{Référence électronique}

Nathalie Bouloux et Odile Redon, «Boccace, « de Canarie et d'autres îles nouvellement découvertes dans l'Océan, au large de l'Espagne » », Médiévales [En ligne], 47 | automne 2004, mis en ligne le 08 décembre 2005, consulté le 22 avril 2022. URL : http://journals.openedition.org/medievales/1598 ; DOI : https://doi.org/10.4000/medievales.1598

Ce document a été généré automatiquement le 22 avril 2022

Tous droits réservés 


\section{Boccace, « de Canarie et d'autres îles nouvellement découvertes dans l'Océan, au large de l'Espagne »}

Traduction du latin et commentaire

Nathalie Bouloux et Odile Redon

Traduction

«L'année de l'incarnation 1341 furent apportées à Florence des lettres de marchands florentins résidant à Séville, cité d'Hispanie Ultérieure, datées du 15 novembre de l'année susdite, et qui contenaient les faits suivants.

Elles disent que le premier juillet de cette année-là deux navires² ${ }^{2}$, affrétés, ainsi qu'un petit navire de guerre, par le roi du Portugal et pourvus par lui des provisions nécessaires à la traversée, partirent de la cité de Lisbonne et firent voile vers le large, avec un équipage composé de soldats florentins, génois, et espagnols ainsi que d'autres Espagnols, emportant en outre des chevaux, des armes et diverses machines de guerre construites pour prendre les cités et les places-fortes. Ils partaient à la recherche de ces îles que nous disons communément "découvertes"; un vent favorable les y amena en 5 jours. Ils ne revinrent à bon port qu'en novembre, ramenant en même temps avec eux : d'abord quatre hommes pris parmi les habitants de ces îles, puis un grand nombre de peaux de boucs et de chèvres, du suif, de l'huile de poisson, des peaux de phoques, du bois rouge qui colore presque comme le brésil - même si les experts en la matière affirment que ce n'est pas du brésil - et aussi des écorces d'arbres qui servent également à teindre en rouge, comme de la terre rouge et d'autres produits du même genre.

3 Le Génois Niccolò de Recco, l'un des commandants de ces navires, interrogé, disait que depuis la cité de Séville jusqu'aux dites îles il y a presque neuf cent mille pas ${ }^{3}$, mais que, depuis le lieu appelé aujourd'hui Cap Saint-Vincent, la distance est bien moins grande. La première des îles découvertes a presque cent cinquante mille pas de circonférence ${ }^{4}$; elle est entièrement rocheuse et forestière, mais abondamment peuplée de chèvres et d'autres animaux ainsi que d'hommes et de femmes nus, au genre de vie et aux usages 
frustes. Il ajoutait que c'est en cette île que lui et ses compagnons avaient pris la plus grande partie des fourrures et du suif, et qu'ils n'avaient pas osé pénétrer trop avant dans l'île.

4 De là, se dirigeant vers une autre île presque plus grande que la précédente, ils virent sur le rivage venir à leur rencontre un grand nombre de gens, hommes et femmes, tous presque nus; certains cependant, qui paraissaient plus importants que les autres, portaient des peaux de chèvre teintes en rouge et en jaune, et, comme on pouvait en juger de loin, très fines et très souples, cousues assez habilement avec des boyaux; et comme on pouvait en juger à leur comportement, il semblait qu'ils eussent un chef, à qui ils montraient tous révérence et respect. Cette foule de gens manifestait son désir d'avoir et entretenir commerce avec ceux qui étaient dans les bateaux. Mais par prudence, lorsque des chaloupes, partant des navires, s'approchèrent du rivage, leurs occupants, ne comprenant absolument pas la langue des insulaires, n'osèrent pas accoster. C'est cependant, rapportent-ils, une manière de parler assez élégante et coulante comme l'italienne. Voyant que personne ne descendait des bateaux, certains tentèrent de les atteindre en nageant, et quelques-uns furent pris : parmi eux, ceux qui furent ramenés ${ }^{5}$.

5 Ensuite, comme ils ne découvraient plus rien d'utile ici, les marins repartirent. En faisant le tour de l'île, ils trouvèrent que sa partie septentrionale était bien plus cultivée que sa partie australe, y voyant de nombreuses habitations, des figuiers et des arbres et des palmiers - bien que stériles ${ }^{6}$, et des jardins potagers, des choux, des légumes. Pour cette raison, ils débarquèrent vingt-cinq marins en armes, qui, fouillant les maisons, y découvrirent environ trente hommes tout nus qui, terrifiés à la vue des armes, s'enfuirent aussitôt. Entrant dans les maisons, ils virent qu'elles étaient faites de pierres équarries remarquablement taillées, et couvertes de bois gigantesques et très beaux; comme les portes étaient fermées et qu'ils voulaient voir l'intérieur, ils se mirent à briser les portes avec des pierres, provoquant la colère de ceux qui fuyaient, qui se mirent à remplir les lieux de hauts cris. Cependant, une fois les serrures fracturées, ils entrèrent dans presque toutes les maisons, mais ils n'y trouvèrent rien d'autre que des figues sèches dans des petits paniers de palmes, bonnes comme celles que nous trouvons à Césène, du froment beaucoup plus beau que le nôtre, aux grains plus longs et plus gros, et très blanc; et aussi de l'orge et d'autres céréales dont, conclurent-ils, se nourrissaient les habitants. Les maisons, outre qu'elles étaient très belles et couvertes d'un très beau bois, étaient à l'intérieur si blanches qu'on les aurait dites blanchies à la chaux. Ils découvrirent également un oratoire ou un temple à l'intérieur duquel ne se trouvait aucune image ni aucun ornement, sauf une seule statue, sculptée dans la pierre, qui représentait un homme tenant un globe dans la main, nu, ses parties honteuses couvertes d'un pagne de palmes, selon leur coutume. Ils la prirent et la chargèrent sur leur bateau pour l'apporter à Lisbonne au retour. Cette île est densément peuplée et exploitée; les habitants récoltent du froment, des céréales, des fruits et surtout des figues. Mais ils mangent le froment et les autres céréales à la manière des oiseaux, ou bien ils fabriquent de la farine, qu'ils mangent cependant sans faire de pain, en buvant de l'eau.

6 S'éloignant de cette île, les marins, qui discernèrent beaucoup d'autres îles, distantes de cinq, dix, vingt ou quarante mille pas, naviguèrent vers une troisième, dans laquelle ils ne trouvèrent rien d'autre que de nombreux arbres, grands et dressés droit vers le ciel. 
7 De là naviguant vers une autre île, ils y trouvèrent abondance de ruisseaux et d'eaux limpides, et là encore beaucoup de bois et des pigeons qu'ils prenaient avec des bâtons et des pierres et qu'ils mangeaient. Ils les disent plus gros que les nôtres et semblables de goût ou même meilleurs. Là aussi ils virent de nombreux faucons et d'autres oiseaux rapaces. Mais ils n'entrèrent pas très avant dans cette île car elle leur sembla complètement déserte.

De là cependant ils découvrirent devant eux une autre île formée de montagnes rocheuses extrêmement hautes, le plus souvent couvertes de nuages, où les pluies sont fréquentes. Mais par temps clair elle est très belle et ceux qui l'ont vue la croient habitée.

9 De là ils abordèrent plusieurs autres îles, au nombre de treize, certaines habitées, d'autres complètement désertes, et plus ils avançaient plus ils en découvraient ; près d'elles la mer est beaucoup plus calme qu'elle ne l'est sur nos rivages et bien que le fond soit propice à l'ancrage il s'y trouve peu de ports. L'eau abonde dans toutes ces îles. Il apparaît que les six îles qu'ils trouvèrent habitées, sur les treize qu'ils abordèrent, sont très peuplées mais elles ne le sont pas également car les unes ont plus d'habitants que les autres. En outre ils disent que les manières de parler sont si différentes entre les îles que de l'une à l'autre ils ne se comprennent absolument pas. De plus il n'existe aucune embarcation ou autre instrument pour passer d'une île à l'autre, à moins d'aller à la nage.

10 Ils découvrirent en outre une autre île où ils ne descendirent pas car il s'y voit un prodige. Ils disent en effet qu'il y existe un mont dont la hauteur est estimée à trente mille pas au moins ${ }^{7}$; on le voit de très loin et quelque chose de blanc apparaît au sommet. Et alors que toute la montagne est de pierre, ce blanc paraît avoir l'aspect d'une citadelle: ils pensent cependant qu'il ne s'agit pas d'une citadelle mais d'une roche très pointue qui semble surmontée d'un mât de la même hauteur que le mât d'un navire, auquel est suspendue l'antenne avec la voile de la grande nef latine creusée en forme d'écu, qui, tirée vers le haut, se gonfle de vent et se déploie largement. Et puis peu à peu on la voit tomber et de la même manière le mât, comme celui d'un navire, et enfin elle remonte et ainsi de suite; ceux qui ont fait le tour de l'île ont observé ce phénomène de tous les côtés. Croyant qu'il résultait d'incantations magiques, ils n'osèrent pas débarquer sur l'île.

11 Ils découvrirent bien d'autres choses que Niccolò n'a pas voulu raconter. Il est clair cependant que ces îles ne sont pas riches puisque les marins ont à peine réussi à rentrer dans leurs frais. Les quatre hommes qu'ils ont ramenés, jeunes, encore imberbes, ont un beau visage et marchent nus. Ils ont cependant un pagne ainsi fait : ils ceignent les reins d'une corde d'où pendent des tiges de palme ou de jonc très serrées, longues d'une paume, ou de deux au plus; celles-ci couvrent le pubis et les parties honteuses par devant et par derrière à moins que le vent ou autre aléa ne les soulève. Ils ne sont pas circoncis; ils ont les cheveux blond-roux, ils les portent longs presque jusqu'au nombril et ils s'en couvrent, tout en marchant pieds nus.

12 L'île où ils ont été enlevés est appelée Canarie et elle est plus peuplée que les autres. Ces hommes ne comprennent absolument aucune manière de parler, bien qu'on ait essayé de s'adresser à eux en beaucoup de langues différentes. Ils ne sont pas plus grands que nous, bien membrés, hardis et forts et, semble-t-il, de grande intelligence. On leur parle par signes et eux-mêmes répondent par signes comme le font les muets. Ils se respectaient l'un l'autre, l'un d'entre eux, il est vrai, plus que tous les autres, et celui-ci 
a un pagne de palmes - alors que les autres l'ont en jonc - colorées en jaune et en rouge. Ils chantent harmonieusement et dansent presque à la française. Ils sont rieurs et vifs et assez tranquilles, plus que la majorité des Espagnols.

Une fois sur le bateau, ils ont mangé du pain et des figues et le pain leur a plu, bien qu'ils n'en aient jamais mangé auparavant. Ils refusent complètement le vin et boivent de l'eau. Ils mangent le froment comme l'orge à pleines mains, du fromage et de la viande, dont il y a très grande abondance et de bonne qualité. Cependant ils n'ont pas de bovins, ni chameaux ni ânes mais ils ont beaucoup de chèvres, de brebis et de sangliers. On leur a fait voir des pièces d'or et d'argent, qui leur étaient complètement inconnues. De même ils ne connaissent aucune sorte d'aromates. Si on leur fait voir des bijoux en or, des vases ciselés, des épées ou des glaives, on a l'impression qu'ils n'en ont jamais vu ni possédé.

montrent un sens extrêmement fort de la sincérité et de la justice; si en effet on donne à l'un d'eux quelque chose à manger, il n'en goûtera pas le moindre morceau avant de l'avoir équitablement divisé et partagé avec les autres.

Leurs femmes se marient et celles qui ont connu des hommes portent des pagnes comme les hommes alors que les vierges vont complètement nues sans éprouver aucune honte à aller ainsi.

comme nous des nombres, faisant passer les unités avant les dizaines de la manière suivante :

1. vait

2. smetti

3. amelotti

4. acodetti

5. simusetti

6. sesetti

7. satti

8. tamatti

9. aldamorana

10. marava

11. vait marava

12. smatta marava

13. amierat marava

14. acodat marava

15. simusat marava

16. sesatti marava.»

Commentaire

L'archipel des Canaries, constitué de sept îles principales et de quelques îlots, situé au large du Sahara occidental, constitue une étape importante des découvertes européennes dans l'Océan atlantique. Son invention est attribuée au génois Lanzarotto Malocello, qui accosta l'île - ensuite appelée Lanzarote - à une date controversée, vraisemblablement peu avant $1336^{8}$. Une expédition affrétée par le roi du Portugal fut lancée en 1341, suivie d'une seconde, organisée cette fois par les Catalans. En 1344, 
Clément VI attribua les Canaries à un prince franco-espagnol, Louis de la Cerda, qui meurt en 1348 sans avoir pris possession de son royaume d'outre-mer. Entre temps, Alphonse VI, roi du Portugal, avait protesté en faisant valoir que les Portugais avaient été les premiers à aborder les Canaries et que, étant donné la situation géographique de l'archipel, ils étaient naturellement les mieux à même d'en assurer la christianisation pour la plus grande gloire de Dieu. Mais après la mort de Louis de la Cerda, plus personne ne réclama les îles et c'est seulement à la fin du XIV ${ }^{e}$ siècle que de nouvelles expéditions portugaises conduisirent à reprendre l'exploration, qui fut suivie au début $\mathrm{du} \mathrm{XV}^{\mathrm{e}}$ siècle par une mise en exploitation anarchique, sous la coupe de deux Français, le sire de Béthencourt et Gadifer de la Salle. Au cours du XVe siècle, la présence européenne puis espagnole eut pour conséquence la disparition totale de la population autochtone, les Ganches, et de leur culture.

Le texte dont nous présentons la traduction française est la version latine par Boccace d'une ou plusieurs lettres par lesquelles des marchands florentins établis à Séville faisaient à leurs associés (compagnie des Bardi ?) le compte-rendu de l'expédition de 1341, en se fondant sur le témoignage d'un des chefs de l'expédition, Niccolò de Recco. L'adaptation réalisée par Boccace se trouve dans un de ses manuscrits de travail, le Zibaldone Magliabechiano (Florence, B. N., Banco Rari, 50, fo 123v-124r), où il copiait au gré de ses intérêts et sans plan préconçu des textes qui l'intéressaient et qui étaient susceptibles de nourrir sa production littéraire et savante ${ }^{9}$. Ce texte a suscité depuis longtemps l'intérêt des historiens et des spécialistes de Boccace ${ }^{10}$. En effet, décrivant le premier contact entre les Européens et les Ganches, il donne des informations uniques sur la culture ganche et, à travers le triple filtre du compte-rendu oral (et peut-être également écrit) de Niccolò de Recco, de la lettre des marchands florentins et de la traduction, il permet d'observer la mise en place des attitudes intellectuelles et économiques qui constituent le fondement des grandes découvertes.

L'expédition décrite dans le texte avait été préparée à partir des récits des campagnes précédentes, dans un double objectif de conquête et d'évaluation économique; elle a duré quatre mois et demi, entre juillet et novembre. Une première séquence du texte, la plus longue, suit le parcours des découvreurs, jusqu'à la petite phrase sur les réserves (politico-économiques?) de Niccolò de Recco. La seconde est une réflexion sur les résultats de l'entreprise.

La première séquence épouse le regard du marin et propose, suivant les usages descriptifs de la géographie médiévale, des mesures précises de distance (1 $300 \mathrm{~km}$ entre Séville et les îles) et de circonférence ( $200 \mathrm{~km}$ pour la première île), une précision que donne aussi fréquemment le Milione de Marco Polo pour décrire les îles; l'œil du marin est aussitôt doublé de celui du marchand, exercé à chercher des produits "utiles» tels que peaux ou graisses... et à les évaluer: voir la discussion sur les colorants rouges. À plusieurs reprises est exprimée la crainte des explorateurs, peur d'accoster, de s'éloigner du rivage, d'une terre sans hommes, d'un phénomène inexpliqué - et dans le cas de "l'île magique », la mesure proposée sort carrément de la raison des nombres (une hauteur de 30000 pas est évidemment impossible). L'activité volcanique est assimilée à un prodige, signe d'une nature dont les lois peuvent se révéler étranges en ces confins du monde.

On se fait comprendre en proposant une comparaison explicite avec les données européennes - un procédé familier des récits de voyage : les figues sèches sont aussi 
bonnes que celles de Césène, le froment est «beaucoup plus beau que le nôtre » mais dans ces îles on ne l'utilise pas pour faire du pain.

Au parcours différencié des lieux, île après île, s'ajoutent des commentaires sur l'occupation de ces lieux par les hommes et sur la rencontre des Espagnols et des Italiens avec cette autre humanité. Ils vont nus ou à peine vêtus de peaux de chèvre colorées; il existe entre eux une hiérarchie; ils parlent une belle langue mais incompréhensible; ils désirent la rencontre mais ils ont peur des armes; ils ne voyagent pas entre une île et l'autre ni ne se comprennent. Ils cultivent soigneusement la terre et vivent dans de belles maisons bien construites, alliant la pierre et le bois, des lieux de vie privée dont le viol les indigne. De leur religion on n'a saisi qu'une statue de pierre anthropomorphe; réciproquement le désir d'évangélisation et le jugement moral sur leur mode de vie sont absents. L'attention du récit au comportement des insulaires répond peut-être à un questionnement utilitaire aux fins de la conquête ; elle laisse aussi entrevoir le Boccace narrateur des nouvelles.

Le bilan présenté dans la séquence finale constate le grand nombre des îles - ouverture sur d'autres découvertes - et leur pauvreté, dont la conclusion logique serait l'inutilité d'une conquête - puisque les insulaires ignorent l'or et les épices - mais aussi sa facilité puisqu'ils ignorent les armes. Par contre il manifeste un grand intérêt anthropologique, avec un retour sur les observations de la première séquence concernant le vêtement, la langue, l'expression de la distinction sociale, les usages alimentaires. Il montre à l'évidence que communication il y a eu entre les insulaires (au moins ceux qui avaient été enlevés) et des hommes d'Europe qui, au moment où s'ouvraient les voies de l'humanisme, s'inquiétaient de connaître les pratiques matrimoniales, l'aire et les usages de vie commune, les systèmes de valeur, le sens moral, la manière de parler et de compter de l'Autre.

\section{NOTES}

1. De Canaria et insulis reliquis ultra Ispaniam in Oceano noviter repertis, M. Pastore Stocchi éd., Milan, 1992 (Tutte le opere di Giovanni Boccaccio, V, 1), p. 970-986.

2. Dans le manuscrit autographe de Boccace (Zibaldone Magliabechiano, Florence, B.N., Banco Rari 50, fo $123 \mathrm{v}^{\circ}$ ), une note marginale indique : Florentinus qui cum hiis navibus prefuit vocatus est Angelinus del Tegghia de Corbizzis consobrinus filiorum Gherardini Giannis. Diverses interprétations de cette note ont été proposées. M. Pastore Stocchi (éd. cit., n. 2, p. 983) la comprend ainsi : « Le Florentin qui eut le commandement s'appelle Angelino del Tegghia dei Corbizzi, cousin des fils de Gherardino Gianni », à la différence de G. Padoan (« Boccaccio e la scoperta delle Canarie », Italia medioevale e umanistica, 7, 1964, p. 267-268) : « Le Florentin qui avec eux - c'est-à-dire avec Niccolò de Recco et le commandant du navire de guerre portugais - commanda le navire... ».

3. Environ $1300 \mathrm{~km}$.

4. Environ $200 \mathrm{~km}$. 
5. La fin de la phrase n'est pas parfaitement claire : Qui tamen, videntes quod nulli ex navibus descendebant, aliqui natantes ad eos pervenire conati sunt, ex quibus quosdam cepere: et ex iis sunt quos aduxerunt (éd. cit., p. 972).

6. Ce que Boccace désigne par l'expression dato steriles palme n'est pas très clair.

7. Le mont en question pourrait être le pic Teyde $(3707 \mathrm{~m})$, volcan qui domine l'île de Tenerife.

8. Ch. Verlinden, «Lanzarotto Molocello et la découverte portugaise des Canaries », Revue belge de philologie et d'histoire, 36, 1958, p. 1173-1209.

9. Il mentionne les données de cette lettre dans son commentaire sur la Divine Comédie (Esposizioni sopra la Commedia di Dante, G. Padoan éd., Tutte le opere di Giovanni Boccaccio, VI, Milan, 1965, p. 334).

10. Entre autres : M. Pastore Stocchi, « Il "De Canaria" Boccaccesco e un locus deperditus nel "De insulis" di Domenico Silvestri », Rinascimento, 10, 1959, p. 143-156 ; G. Padoan, " Boccaccio e la scoperta delle Canarie », Italia medioevale e umanistica, 7, 1964, p. 263-277.

\section{AUTEURS}

NATHALIE BOULOUX

Université François Rabelais, UFR Arts et sciences humaines, Département d'Histoire, 3 , rue des Tanneurs, BP 4103, F-37041 Tours Cedex 01

\section{ODILE REDON}

Université Paris VIII, 2, rue de la Liberté, Département d'Histoire, F-93526 Saint-Denis Cedex 